\title{
Inflammation, age and changing microbiology: the search for causation in the cystic fibrosis airways
}

\author{
Geraint B. Rogers ${ }^{1,2}$ \\ Affiliations: 'South Australian Health and Medical Research Institute, Adelaide, Australia. ${ }^{2}$ SAHMRI \\ Microbiome Research Laboratory, School of Medicine, Flinders University, Adelaide, Australia. \\ Correspondence: Geraint B. Rogers, School of Medicine, Flinders University, University Drive, Bedford Park, \\ Adelaide SA 5042, Australia. E-mail: geraint.rogersasahmri.com
}

@ERSpublications

A survey of CF BALF reports lung microbiota to vary with age and inflammation but causality remains hard to pin down http://ow.ly/hEjX30fVMoL

Cite this article as: Rogers GB. Inflammation, age and changing microbiology: the search for causation in the cystic fibrosis airways. Eur Respir J 2017; 50: 1701935 [https://doi.org/10.1183/13993003.01935-2017].

Since the first reports of bacterial lung infections in patients with cystic fibrosis (CF) almost 80 years ago, concerted efforts have been made to identify the microbes responsible and to determine their contribution to lung disease. However, while CF respiratory microbiology has helped to shape clinical practice, our understanding of the relationships between microbial colonisation of the lung, inflammation and disease remains relatively poor.

The development of culture-independent analytical techniques, such as 16S ribosomal RNA (rRNA) gene amplicon sequencing, has provided a means to characterise CF airway microbiology more comprehensively. The widespread application of such approaches has revealed that the small group of CF airway-associated microbes is in fact part of a larger and more complex CF lung microbiota [1-3]. In particular, microbes that are able to exploit lower oxygen tensions, including members of the Prevotella, Veillonella, Streptococcus and Gemella genera, have been reported consistently in CF lower airway samples [1, 4-8]. While analysis of diseased lung tissue obtained directly through surgery [9], and the comparative analysis of upper and lower airway samples [10], suggest lung colonisation by these taxa, there is an ongoing debate around their clinical significance. Little evidence yet exists that this wider lung microbiota contributes directly to inflammation, although intriguingly, studies in animal models have demonstrated that some oropharyngeal anaerobes can significantly increase the expression of Pseudomonas aeruginosa pathogenicity traits when present as co-infections [11, 12].

To date, efforts to understand the relationships between the composition of the CF lung microbiota and clinical and immunological measures have mostly taken the form of single-centre, cross-sectional studies. Despite being based typically on small and heterogeneous patient populations, these studies have reported remarkably consistent findings. One notable observation has been that the composition of the CF lung microbiota changes with increasing age, exposure to antimicrobials and disease severity [2, 13]. This evolving composition represents a transition from a diverse, but relatively low abundance, microbial community in infants, where taxa such as Streptococcus, Prevotella and Veillonella are particularly prevalent, to an increasingly low diversity, high bacterial load community in older individuals, often dominated by $P$. aeruginosa [14]. Indeed, by end-stage disease, the predominance of pathogens, such as

Received: Sept 232017 | Accepted: Sept 252017

Conflict of interest: Disclosures can be found alongside this article at erj.ersjournals.com

Copyright OERS 2017 
P. aeruginosa, can become almost complete [15]. However, whether these microbiological changes influence disease progression, or are simply the result of a changing lung environment and an increasing exposure to antibiotics, remains unclear.

The study by ZEMANICK et al. [16] in this issue of the European Respiratory Journal (ERJ) is a further attempt to relate CF lung microbiota composition to patient characteristics and disease. The analysis was based on the application of $16 \mathrm{~S}$ rRNA amplicon sequencing to bronchoalveolar fluid (BALF) samples obtained during clinically indicated bronchoscopy in both paediatric and adult patient populations. In total, samples from $146 \mathrm{CF}$ patients and 45 disease controls, drawn from 13 separate centres across the USA, were assessed, making this the largest BALF-based analysis of its kind to date.

Much of what Zemanick et al. [16] report corroborates the findings of previous, smaller investigations. For example, they found that the airway microbiota composition in patients $<2$ years of age is dominated, in many cases, by Streptococcus, Prevotella or Veillonella. In contrast, older patients exhibited higher levels or airway inflammation, decreased microbial diversity and a greater abundance of recognised CF pathogens. Overall, they report that "nontraditional taxa" were the dominant taxon in one in five of the CF BALF samples assessed, while no sequence data were generated from $20 \%$ of CF adults and $44 \%$ of CF children (a phenomenon the authors attribute to low bacterial load).

It is interesting to speculate about what this changing microbiological landscape might represent. The prevalence of Streptococcus, Veillonella and Prevotella in lower airway samples, particularly from paediatric patients, might simply be the result of ongoing translocation of microbes from the upper airways, with relative abundance of these taxa appearing high due to the low total bacterial load. Alternatively, the detection of these taxa may represent true colonisation of the CF lung. In a recent study published in the ERJ, PREvaes et al. [17] compared microbiota composition in BALF with that in nasopharyngeal and oropharyngeal samples from infants with CF. The authors reported inconsistent intraindividual concordance between microbiota of the upper and lower respiratory niches, suggesting that the lungs of infants with CF may have their own microbiome, seeded by, but not identical to, the upper respiratory tract microbiome.

If nontraditional taxa do colonise the CF lower airways, whether they contribute directly to airway inflammation is uncertain. ZEMANICK et al. [16] point out that patients whose airway microbiota was characterised by a high relative abundance of these taxa had higher levels of airway inflammation than disease controls, although whether this stems from the presence of microbes or from the direct effects of underlying pathophysiology was not investigated.

Whether nontraditional taxa might influence the risk of infection by recognised CF pathogens, such as $P$. aeruginosa, is also not known. It is certainly possible that the lung microbiota contributes to the creation of an airway environment that is more or less conducive to pseudomonal growth, as has been demonstrated in animal models for other airway pathogens [18]. Potentially, such an interaction might contribute to the failure of a subset of CF patients to ever acquire chronic $P$. aeruginosa infections, despite the environmental prevalence of this pathogen. Conversely, the development of infection by species associated with poor clinical outcomes, such as P. aeruginosa, is likely to also affect the composition of the wider lung microbiota. Increases in inflammation and antimicrobial exposure that are associated with $P$. aeruginosa infection are likely to lead to a constriction of microbial diversity, while the predominance of a single species will have the effect of pushing down the relative abundance of other taxa. Despite this, high density pseudomonal growth and poor clearance of airway secretions will contribute to the development of regions of low oxygen tension that support colonisation by anaerobic species, whose fermentation products have the potential not only to damage the airways directly [19] but also to upregulate the expression of pseudomonal pathogenicity traits [20].

The strategy employed by ZемANick et al. [16], and others, to gain insight into the relationship between airway microbiology and factors such as age has been to assess samples from as large a population as possible, with subsequent stratification according to clinical or demographic measures. An alternative, but potentially complementary, approach is to perform longitudinal analysis of individual patients. Such a strategy enables the relative timing of changes in microbiology and clinical measures to be assessed, as well as a more ready separation of chronological age from each patient's unique treatment history and disease characteristics. Interestingly, a recent study by FrAYMAN et al. [5], involving 16S rRNA amplicon sequencing was performed on BALF collected longitudinally from 48 paediatric CF patients, reported transitions in airway microbiology similar to those described by ZEMANicK et al. [16], despite considerable intersubject variability.

ZeMANICK et al. [16] also report disparities between sequencing- and culture-derived data, including in the detection of species that are commonly isolated through standard diagnostic microbiology. Pseudomonas, 
for example, was detected in five culture-negative patients at low relative abundance (0.006-1.13\%). While this finding appears to be a common feature of sequencing-based airway studies, the appropriate clinical response is debatable. One interpretation, suggested by the authors, is that molecular detection represents an early indication of potential infection and provides an opportunity for intervention. However, exposure to environmental sources of $P$. aeruginosa is common and its presence in the lower airways at low abundance might be transient. Indeed, transient $P$. aeruginosa colonisation is not uncommon in paediatric CF patients and is not, in itself, associated with a reduction in pulmonary function [21]. The strong association between chronic infection and poor clinical outcomes provides a compelling argument for eradication at first detection [22, 23], although retrospective analysis suggests that that there is little difference in rates of recurrence of pseudomonal infection between those who received antibiotics during the initial therapy periods and those who did not [24]. The fact that species such as $P$. aeruginosa, as well as the Burkholderia and Strenotrophomonas genera, are common sequencing artefacts due to reagent contamination [25] suggests these findings require careful consideration.

Interestingly, the authors detected Bordetella in three paediatric CF BALF samples, at $>50 \%$ relative abundance in each case, from patients experiencing pulmonary exacerbation. Bordetella pertussis and Bordetella bronchiseptica (predominantly an animal pathogen) are both capable of causing chronic infection in CF patients $[26,27]$, but their slow rates of growth mean that they can go undetected in mixed respiratory cultures. The use of targeted, culture-independent assays, such as those based on quantitative PCR, should therefore be considered, and might provide a useful bridge between sequencing-derived data and routine diagnostics.

The study also highlights an important limitation of 16S rRNA gene sequencing: its inability to consistently provide species-level bacterial identification. This weakness is particularly problematic when trying to assess the clinical significance of nontraditional taxa. For example, Streptococcus, a genus commonly reported in CF lung samples by sequencing, is diverse, containing species that differ substantially in their pathogenic potential. Building on the work of ZEMANICK et al. [16] and others, it is now important that higher resolution analytical techniques, whether sequence- or culture-based, are applied to the analysis of CF lung. Such strategies would not only clarify the overlap between upper respiratory microbiota and the composition of lower airway samples, but would potentially inform our understanding of mechanisms by which these taxa might influence CF lung disease.

\section{References}

1 Rogers GB, Carroll MP, Serisier DJ, et al. Characterization of bacterial community diversity in cystic fibrosis lung infections by use of $16 \mathrm{~S}$ ribosomal DNA terminal restriction fragment length polymorphism profiling. $J$ Clin Microbiol 2004; 42: 5176-5183.

2 Zhao J, Schloss PD, Kalikin LM, et al. Decade-long bacterial community dynamics in cystic fibrosis airways. Proc Natl Acad Sci USA 2012; 109: 5809-5814.

3 Stressmann FA, Rogers GB, van der Gast CJ, et al. Long-term cultivation-independent microbial diversity analysis demonstrates that bacterial communities infecting the adult cystic fibrosis lung show stability and resilience. Thorax 2012; 67: 867-873.

4 Laguna TA, Wagner BD, Williams $\mathrm{CB}$, et al. Airway microbiota in bronchoalveolar lavage fluid from clinically well infants with cystic fibrosis. PLoS One 2016; 11: e0167649.

5 Frayman KB, Armstrong DS, Carzino R, et al. The lower airway microbiota in early cystic fibrosis lung disease: a longitudinal analysis. Thorax 2017; in press [https:/doi.org/10.1136/thoraxjnl-2016-209279].

6 Carmody LA, Zhao J, Schloss PD, et al. Changes in cystic fibrosis airway microbiota at pulmonary exacerbation. Ann Am Thorac Soc 2013; 10: 179-187.

7 Coburn B, Wang PW, Diaz Caballero J, et al. Lung microbiota across age and disease stage in cystic fibrosis. Sci Rep 2015; 5: 10241.

8 Fodor AA, Klem ER, Gilpin DF, et al. The adult cystic fibrosis airway microbiota is stable over time and infection type, and highly resilient to antibiotic treatment of exacerbations. PLoS One 2012; 7: e45001.

9 Brown PS, Pope CE, Marsh RL, et al. Directly sampling the lung of a young child with cystic fibrosis reveals diverse microbiota. Ann Am Thorac Soc 2014; 11: 1049-1055.

10 Rogers GB, Carroll MP, Serisier DJ, et al. Use of $16 \mathrm{~S}$ rRNA gene profiling by terminal restriction fragment length polymorphism analysis to compare bacterial communities in sputum and mouthwash samples from patients with cystic fibrosis. J Clin Microbiol 2006; 44: 2601-2604.

11 Duan K, Dammel C, Stein J, et al. Modulation of Pseudomonas aeruginosa gene expression by host microflora through interspecies communication. Mol Microbiol 2003; 50: 1477-1491.

12 Sibley CD, Duan K, Fischer C, et al. Discerning the complexity of community interactions using a Drosophila model of polymicrobial infections. PLoS Pathog 2008; 4: e1000184.

13 Klepac-Ceraj V, Lemon KP, Martin TR, et al. Relationship between cystic fibrosis respiratory tract bacterial communities and age, genotype, antibiotics and Pseudomonas aeruginosa. Environ Microbiol 2010; 12: 1293-1303.

14 Rogers GB, Hoffman LR, Carroll MP, et al. Interpreting infective microbiota: the importance of an ecological perspective. Trends Microbiol 2013; 21: 271-276.

15 Goddard AF, Staudinger BJ, Dowd SE, et al. Direct sampling of cystic fibrosis lungs indicates that DNA-based analyses of upper-airway specimens can misrepresent lung microbiota. Proc Natl Acad Sci USA 2012; 109: 13769-13774. 
16 Zemanick ET, Wagner BD, Robertson CE, et al. Airway microbiota across age and disease spectrum in cystic fibrosis. Eur Respir J; 50: 1700832.

17 Prevaes SM, de Steenhuijsen Piters WA, de Winter-de Groot KM, et al. Concordance between upper and lower airway microbiota in infants with cystic fibrosis. Eur Respir J 2017; 49: 1602235.

18 Weyrich LS, Feaga HA, Park J, et al. Resident microbiota affect Bordetella pertussis infectious dose and host specificity. J Infect Dis 2014; 209: 913-921.

19 Whiteson KL, Meinardi S, Lim YW, et al. Breath gas metabolites and bacterial metagenomes from cystic fibrosis airways indicate active $\mathrm{pH}$ neutral 2,3-butanedione fermentation. ISME J 2014; 8: 1247-1258.

20 Venkataraman A, Rosenbaum MA, Werner JJ, et al. Metabolite transfer with the fermentation product 2,3-butanediol enhances virulence by Pseudomonas aeruginosa. ISME J 2014; 8: 1210-1220.

21 Amin R, Lam M, Dupuis A, et al. The effect of early Pseudomonas aeruginosa treatment on lung function in pediatric cystic fibrosis. Pediatr Pulmonol 2011; 46: 554-558.

22 Emerson J, Rosenfeld M, McNamara S, et al. Pseudomonas aeruginosa and other predictors of mortality and morbidity in young children with cystic fibrosis. Pediatr Pulmonol 2002; 34: 91-100.

23 Frederiksen B, Koch C, Hoiby N. Antibiotic treatment of initial colonization with Pseudomonas aeruginosa postpones chronic infection and prevents deterioration of pulmonary function in cystic fibrosis. Pediatr Pulmonol 1997; 23: 330-335.

24 Mayer-Hamblett N, Rosenfeld M, Treggiari MM, et al. Standard care versus protocol based therapy for new onset Pseudomonas aeruginosa in cystic fibrosis. Pediatr Pulmonol 2013; 48: 943-953.

25 Salter SJ, Cox MJ, Turek EM, et al. Reagent and laboratory contamination can critically impact sequence-based microbiome analyses. BMC Biol 2014; 12: 87.

26 van den Brink G, Wishaupt JO, Douma JC, et al. Bordetella pertussis: an underreported pathogen in pediatric respiratory infections, a prospective cohort study. BMC Infect Dis 2014; 14: 526.

27 El Khatib N, Ferroni A, Le Bourgeois M, et al. Persistent Bordetella bronchiseptica infection in a child with cystic fibrosis: relationship to bacterial phenotype. J Cyst Fibros 2015; 14: E13-E15. 